\title{
Cannabis Responsive Head Injury Induced Mutiple Disabilities: A Case Report
}

\section{Manju Sharma}

Prostate Centre at VGH \& Green Cross Society of BC, Vancouver, Canada.

Email: msharma@prostatecentre.com

Received October $2^{\text {nd }}, 2011$; revised November $14^{\text {th }}, 2011$; accepted December $19^{\text {th }}, 2011$

\begin{abstract}
In recent years cannabinoids and their derivatives have drawn renewed attention because of their diverse pharmacologic activities. We report here one such case, where all types of medical \& psychiatric treatment failed to improve the symptoms; however cannabis use was able to bring back this patient to normal productive \& meaningful life. The patient was a 47 year old left handed Caucasian had minor subdural hematoma at the posterior vertex and a minor focal subarachnoid haemorrhage following a physical assault. His impairments included cognitive slowing with decreased short term memory, organized skill \& language deficit. His physical disabilities included spastic gait (hemiplegic), VII nerve palsy, mild cerebellar dysfunction, blurred vision and easy fatigue. He was unable to return to work or drive. In addition to cognitive deficit, right hemi paresis \& dizziness; he had symptoms of anxiety \& depression. Cannabis improved his gait and brought back lots of his memory. Within 6 months all his symptoms abated with use of cannabis and he started to look at cannabis as a real medicine. Slowly he also had improvement in cognitive functions, memory, vocabulary and his gait became increasingly better day by day with continued use of cannabis.
\end{abstract}

Keywords: Cannabinoids; THC; CBD; CBN

\section{Introduction}

Cannabis has been used for treatment in various types of malignancies. Its use in the management of disabilities following moderately severe traumatic brain injuries has not been reported. We report here one such case, where all types of medical \& psychiatric treatment failed to improve the symptoms; however cannabis use was able to bring back this patient to normal productive \& meaningful life.

\section{Case Report}

P Hunt, a 47 year old left handed Caucasian had minor subdural hematoma at the posterior vertex and a minor focal subarachnoid haemorrhage following a physical assault on $24^{\text {th }}$ April 1997. He was hospitalized and had a Glasgow Coma score of 6. He remained unconscious for 3 days. He had post traumatic amnesia \& was discharged on June $27^{\text {th }}$, 1997 with residual numbness in right hand \& toes. He accidentally cut his finger, subsequently. He had occasional chocking with thin fluids. His major problem was cognitive disabilities. He had poor short term memories and was forced to use a memory book. He had some language problem along with blurry vision while watching TV. He also noted subtle changes in per- sonality and felt less inhibited and more prone to outburst of laughing or tears. However he felt a bit more compassionate than before. He also complained of dry skin over face \& scalp. Mr. Hunt was allergic to hay \& codeine. He was a smoker \& a social drinker. He had minimum family support $\&$ had received education up to $12^{\text {th }}$ standard. He was running a small construction company before the assault.

\subsection{Physical Examination}

A fully oriented pleasant man without distress. He was able to recall 3 out of 3 items after 5 minutes \& was able to subtract serial 3 without difficulty. He did well with serial 7 but made gross errors when he reached 50.His forward digit span was 7 and abstraction was relatively intact. He had right supranuclear facial palsy. Extra ocular movements were full \& visual fields were grossly normal. Sensations were intact. Tongue $\&$ palate were in midline. The tone was normal in upper \& lower limbs \& bulk was symmetrical. Power was grade $\mathrm{V}$ on left and it was IV plus on the right side. Posterior column sensations were intact. There was no subjective loss of pinprick in distal phalanges on right side. However he had patch pinprick loss in right foot. He was however able to differentiate between light touch \& pinprick. Deep ten- 
don reflexes were brisk bilaterally; however these were more on right side. Cerebellar functions were slightly impaired on right side. Romberg's test was negative, although there was increased swaying with closed eyes. Gait was consistent with spastic hemiplegic on right side. It was wide based \& somewhat ataxic. There was decreased arm swaying on right side with flexor posturing. He was able to do heel-toe walk but was unable to perform tandem gait. He was able to stand on one leg with eyes closed. Therefore, his impairments included cognitive slowing with decreased short term memory, organized skill \& language deficit. His physical disabilities included spastic gait (hemiplegic), VII nerve palsy, mild cerebellar dysfunction, blurred vision and easy fatigue. He was unable to return to work or drive.

During the outpatient program a number of issues were addressed which included, assistance of finances \& physiotherapy for the gait, balancing \& coordination exercises without much benefit. In October 1998 he was again hospitalized due to complaint of dizzy sensations (vertigo) which was thought to be central in origin for which he was advised compensation techniques. His physical \& cognitive dysfunctions remain unchanged. He continued to have balancing difficulty as he was constantly unsteady \& insecure on his feet. In February 1999, in addition to cognitive deficit \& right hemi paresis, he had symptoms suggestive of depression \& anxiety. He had insomnia \& remained preoccupied with thoughts of his collapse of business and being cheated by his foreman. He had difficulty in talking and described it as "I freeze \& cannot talk" especially when confronted with someone as he had difficulty in thinking quickly. He was therefore put on Effexor but he developed tachycardia \& generalized weakness, therefore medication was discontinued.

\subsection{Mental Status Examination}

Mental assessment was carried out by the psychiatrist. A casually dressed middle aged Caucasian, looked pleasant, co-operative, accessible \& reliable. His speech was normal rate and volume \& was mainly goal oriented. He was preoccupied with the idea of collapse of his business. He described his mood being depressed \& his effect was restricted to the element of sadness, although he was able to smile appropriately at times. In terms of suicidal ideation, he stated that it crossed his mind a couple of times but he was able to resist it. He denied any intent to hurt himself. There was no history of delusions or auditory or visual hallucinations. His insight and judgment seemed fair.

\subsection{Rehabilitation \& Follow Up}

Therefore in addition to cognitive deficit, right hemi pa- resis \& dizziness; he had symptoms of anxiety \& depression. In order to calm his nerves, he was put on Zoloft 25 mg per day which was gradually titrated upwards till 200 $\mathrm{mg} /$ day. For his dizziness, vestibular rehabilitation program was instituted but he made little progress. He was also referred to Trauma Recovery Group for ongoing support, but in vain. Since he did not make any significant progress after his discharge from rehabilitation in 1997, therefore no further follow up were arranged for him. Therefore he weaned himself off of 150 and then to $200 \mathrm{mg}$ Zoloft and Ativan but at the same time he started using Cannabis (with high CBD and low THC concentration). He started feeling that his vocabulary was returning and he could talk to people more easily. He smoked his whole life except after his injury in 1997 and about 1/2 year later when he joined the society who sold cannabis. He smoked quite frequently now. His daily consumption of this cannabis was 7 - 10 grams consumed as baked stuff and smoking. This strain of cannabis improved his gait and brought a lot of his memory back. Within 6 months all his symptoms abated with use of cannabis and he started to look at cannabis as a real medicine. Slowly he had improvement in cognitive functions and his memory, vocabulary and gait became increasingly better day by day. His former corporate knowledge returned and he remembered owning a medium sized construction company. His dizziness slowly abated and he could walk without fear of falling down as he felt more confident and steady. He utilized his previous knowledge of running the construction company and formed a non-profiting society for people with disabilities. Today, Paul is the Founder and President of the Green Cross Society of BC.

\section{Discussion}

The therapeutic properties of the hemp plant, Cannabis sativa, have been known since antiquity, but the recreational use of its euphoric and other psychoactive effects has restricted for a long time research on its possible pharmaceutical application. In recent years cannabinoids and their derivatives have drawn renewed attention because of their diverse pharmacologic activities. In the present case there were four major symptoms; cognitive dysfunction, spasticity, unsteadiness vertigo, blurred vision and anxiety with depression superadded to them. The purpose of this case study was to demonstrate the efficacy and usefulness of the standardized whole plant cannabis as a medicine. Indeed, it is a difficult task of elucidating the efficacy of such preparations.

A significant improvement in cognitive functions, decrease in muscular spasticity, unsteadiness, and dizziness, improvement in symptoms of blurred vision, anxiety, depression and general wellbeing were observed after the 
use of this drug in the present case. Preliminary research on synthetic THC has been conducted in Tourette syndrome and it has been found useful in reducing tics \& a trend towards improving cognitive functioning were reported during and after treatment [1]. A correlation between increase of cognitive functions and cannabinoids use has also been established in schizophrenics [2]. Central cannabinoid CB1 receptors have been found to be located at high concentrations in the output nuclei of the basal ganglia, in forebrain areas associated with higher cognitive functions, in the molecular layers of the cerebellum, hippocampal dentate gyrus, and other parts of the hippocampal formation [3]. After the discovery of the two specific molecular targets for THC, CB1 and CB2 [4], it became clear that most of the effects of marijuana in the brain and peripheral tissues were due to activation of these two G-protein-coupled cannabinoid receptors. Assuming an involvement of the CB1 receptor system in pathophysiology, it can be speculated that improved cognitive functions after cannabinoid treatment were because of a dysfunction in the cannabinoid receptor system following brain injury. Therefore influence of cannabinoids on cognitive processes might be different compared to healthy users. It has also been demonstrated that cannabinoids mediate increases in prefrontal noradrenaline, acetylcholine, and glutamate. In this context, it has been suggested that cannabis use may enhance executive functions and attention/processing speed in schizophrenia by stimulating prefrontal neurotransmission. These findings have important implications for the treatment of cognitive impairment. Functionally, the patient went from a state of virtual incapacitation to a dramatic improvement over the course of one year. The presence of (CBD) Cannabidiol and Cannabinol (CBN) appear to modulate the binding of Delta-9 Tetrahydrocannabinol (THC) to its receptor and thus alter the efficacy of the preparation. The cannabis which was used by this patient had high CBD and low concentration of THC.

Cannabinoids are found to have particular application as neuroprotectants, in limiting neurological damage following ischemic stroke \& trauma. Non-psychoactive cannabinoids, such as cannabidiol, are particularly advantageous to use because they avoid toxicity. Neurogenerative properties of cannabinoids in adult brain cell have been established and new research indicates that the effect comes from non-psychoactive cannabinoids attaching to CB 1 receptors. It has now been shown that CBD in cannabinoids increases the formation of new nerve cells in brains of adult mice without impairing learning, while THC, the primary psychoactive component, has no effect on neurogenesis [5].

It has been found that CBD had anxiolytic and antidepressant properties, while THC analogues were shown to have anti-anxiety effect and potential of treating schizophrenia. Considering that the anxiolytic properties of CBD can be mediated by the activation of 5-HT1A receptors and that this modulation can induce antidepressant effects, this hypothesis has recently been tested using the forced swim test in mice \& CBD was shown to be anxiolytic [6] and antidepressant [7]. It was concluded that cannabinoid receptors modulate a variety of brain functions including anxiety, fear, mood and target endocannabinoid system [8]. THC has other effects like relaxation, euphoria, altered space time perception; alteration of visual, auditory \& olfactory sensations. The improvement in symptoms of anxiety, depression, spastcity \& dizziness in the present case was therefore related to modulation of brain functions. Cannabinoids may, in the future, become an important option in the treatment of psychiatric symptoms and disorders. Due to the absence of psychoactive or cognitive effects, to its safety and tolerability, to the existence of clinical trials with positive results, and to its broad pharmacological spectrum, CBD is possibly the cannabinoid more likely to have initial findings translated into clinical practice. In particular, the results indicating that CBD has anti-psychotic and anxiolytic properties seem to be well established. These effects can be dependent on the cannabinoid dose and on genetic and individual factors that are not currently understood.

\section{Consent}

Written informed consent was obtained from the patient for publication of this case report. A copy of the written consent is available for review by the Editor-in-Chief of this journal.

\section{REFERENCES}

[1] K. R. Muller-Vahl, H. Prevedel, K. Theloe, H. Kolbe, H. M. Emrich and U. Schneider, "Treatment of Tourette syndrome with Delta-9-Tetrahydrocannabinol (D9-THC): No Influence on Neuropsychological Performance,” Neuropsychopharmacology, Vol. 28, 2003, pp. 384-388. doi:10.1038/sj.npp.1300047

[2] C. M. Coulston, M. Perdices and C. C. Tennant, “The Neuropsychological Correlates of Cannabis Use in Schizophrenia Lifetime Abuse/Dependence, Frequency of Use, and Recency of Use," Schizophrenia Research, Vol. 96, No. 1-3, 2007, pp. 169-184. doi:10.1016/j.schres.2007.08.006

[3] M. Herkenham, A. B. Lynn, M. D. Little, M. R. Johnson, L. S. Melvin, B. R. de Costa, et al., "Cannabinoid Receptor Localization in Brain," Proceedings of the National Academy of Sciences of the United States of America, Vol. 87, 1990, pp. 1932-1936.

[4] R. J. McKallip, M. Nagarkatti and P. S. Nagarkatti, "Delta-9-tetrahydrocannabinol Enhances Breast Cancer 
Growth and Metastasis by Suppression of the Antitumor Immune Response,” Journal of Immunology, Vol. 174, No. 6, 2005, pp. 3281-3289.

[5] S. A. Wolf, A. Bick-Sander, K. Fabe, P. Leal-Galicia, et al., "Cannabinoid Receptor CB1 Mediates Baseline and Activity-Induced Survival of New Neurons in Adult Hippocampal Neurogenesis," Cell Communication and Signaling, Vol. 8, 2010, p. 12.

[6] A. W. Zuardi, "Cannabidiol: From an Inactive Cannabinoid to a Drug with Wide Spectrum of Action," Revista Brasileira de Psiquiatria, Vol. 30, No. 3, 2008, pp. 271280. doi:10.1590/S1516-44462008000300015
[7] T. V. Zanelati, C. Biojone, F. A. Moreira, F. S. Guimarães and S. R. Joca, "Antidepressant Like Effects of Cannabidiol in Mice: Possible Involvement of 5-HT1 Areceptor,” British Journal of Pharmacology, Vol. 159, No. 1, 2010, pp. 122-128. doi:10.1111/j.1476-5381.2009.00521.x

[8] V. M. Saito, C. T. Wotjak and F. A. Moreira, "Pharmacological Exploitation of the Endocannabinoid System: New Perspectives for the Treatment of Depression and Anxiety Disorders?” Revista Brasileira de Psiquiatria, Vol. 32, Suppl. 1, 2010, pp. 7-14. 